\title{
Effects of alkoxy substitution on the crystal structure of 2,3-bis[(E)-4- (diethylamino)-2-alkoxybenzylideneamino|fumaronitrile derivatives $\dagger$
}

\author{
Byung-Soon Kim, ${ }^{a}$ Takumi Jindo, ${ }^{b}$ Ryohei Eto, ${ }^{a}$ Yohei Shinohara, ${ }^{a}$ Young-A Son, ${ }^{c}$ Sung-Hoon Kim ${ }^{d e}$ \\ and Shinya Matsumoto*ab
}

Received 12th February 2011, Accepted 24th May 2011

DOI: 10.1039/c1ce05198a

The bisazomethine dyes derived from diaminomaleonitrile and aminobenzaldehydes, 2,3-bis[(E)-4(dialkylamino)benzylideneamino]fumaronitrile derivatives, are known to have potential as dyes for forming J-aggregate vapour-deposited films, even though appropriate conditions for preparation of their pure J-aggregate films are unknown. In this study, an attempt was made to investigate the effects of alkoxy substitution on the crystal structures of eight bisazomethine dyes; our final goal was to obtain pure J-aggregates of bisazomethine dyes in a crystalline state. The focus of this study is on the relationship between changes in the molecular structure and the crystal structure, characterized by a two-dimensional molecular stacking layer and its alignment. We found that the interlayer distance between adjacent two-dimensional stacking layers can be controlled by alkoxy substitution, when the substituent chain length is less than $\mathrm{C} 10$, without any significant structural changes in the stacking layer. Our observation was confirmed by lattice energy calculations from an energetic perspective.

\section{Introduction}

Flexible opto-electronic devices have attracted much attention as a promising future technology for the needs of a highly advanced information society. Organic molecules are considered to be promising materials for flexible device applications such as organic light-emitting diodes, ${ }^{1-4}$ organic thin-film transistors, ${ }^{5,6}$ and organic solar cells. ${ }^{7,8}$ In addition to providing light-weight flexible structures for organic devices, organic molecular materials are low-cost and eco friendly manufacturing processes such as ink-jet printing can be used. ${ }^{9-12}$ Organic molecules are also thought to have great advantages over conventional inorganic compounds because their molecular structures can be easily modified in order to improve their physico-chemical properties. ${ }^{13}$ High-performance organic materials are needed in the development of the aforementioned applications in order to achieve the characteristics required in practical applications. To achieve this objective, the solid-state structures of organic molecules should

${ }^{a}$ Graduate School of Environmental and Information Sciences, Yokohama National University, Hodogaya-ku, Yokohama, 240-8501, Japan. E-mail: smatsu@edhs.ynu.ac.jp; Fax: +81 45339 3345; Tel: +81 453393366

${ }^{b}$ Faculty of Education and Human Sciences, Yokohama National University, Hodogaya-ku, Yokohama, 240-8501, Japan

'School of Chemical and Biological Engineering, Chungnam National University, Daejeon, 305-764, Korea

${ }^{d}$ Department of Textile System Engineering, Kyungpook National University, Daegu, 702-701, Korea

eSchool of Chemical Science \& Technology, Zhanjiang Normal University, Zhanjiang, 524048, PR China

$\dagger$ CCDC reference numbers 811127-811134. For crystallographic data in CIF or other electronic format see DOI: $10.1039 / \mathrm{c} 1 \mathrm{ce} 05198 \mathrm{a}$ be intensively studied, as the history of the development of silicon-based semiconductor technologies indicates. However, it is still very difficult to design organic molecules with the desired solid-state properties.

The J-aggregates ${ }^{14-27}$ of organic dyes can be regarded as potential candidates. The optical features of J-aggregates result from a resonance electronic state within the aggregates, ${ }^{25}$ so $\mathrm{J}$-aggregates are expected to exhibit ultrafast optical responses, ${ }^{22}$ as well as resonance fluorescence, ${ }^{23}$ because there is no coupling of the vibrational and rotational state changes with the electronic transition. This unique electronic nature should be applicable to high-performance device applications, as described above. For solid-film applications, however, we have to find answers to scientific as well as technological issues regarding $\mathrm{J}$-aggregate formation in solids. The optical properties of J-aggregates may originate from specific one-dimensional (D) or 2-D electronic states, ${ }^{25,26}$ based on excitonic resonance interactions. ${ }^{24}$ There have been some theoretical studies ${ }^{21,24,27}$ on these models, and Kato et al. reported experimental observation of the proposed low-D molecular arrangement for J-aggregates by synchrotron

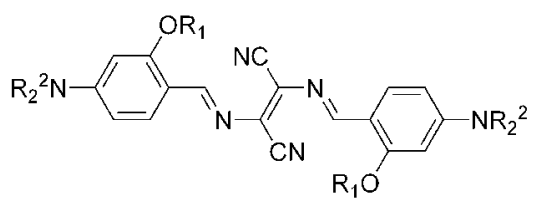

$1 \mathrm{R}_{1}=\mathrm{C}_{6} \mathrm{H}_{5} \mathrm{CH}_{2}, 2 \mathrm{R}_{1}=\mathrm{C}_{6} \mathrm{H}_{5}, 3 \mathrm{R}_{1}=\mathrm{C}_{2} \mathrm{H}_{5}, 4 \mathrm{R}_{1}=\mathrm{C}_{3} \mathrm{H}_{7}$, $5 \mathrm{R}_{1}=\mathrm{C}_{5} \mathrm{H}_{11}, 6 \mathrm{R}_{1}=\mathrm{C}_{7} \mathrm{H}_{15}, 7 \mathrm{R}_{1}=\mathrm{C}_{8} \mathrm{H}_{17}, 8 \mathrm{R}_{1}=\mathrm{C}_{10} \mathrm{H}_{21}$ $\left(\mathrm{R}_{2}=\mathrm{C}_{2} \mathrm{H}_{5}\right)$

Scheme 1 Chemical structures of dyes 1-8. 


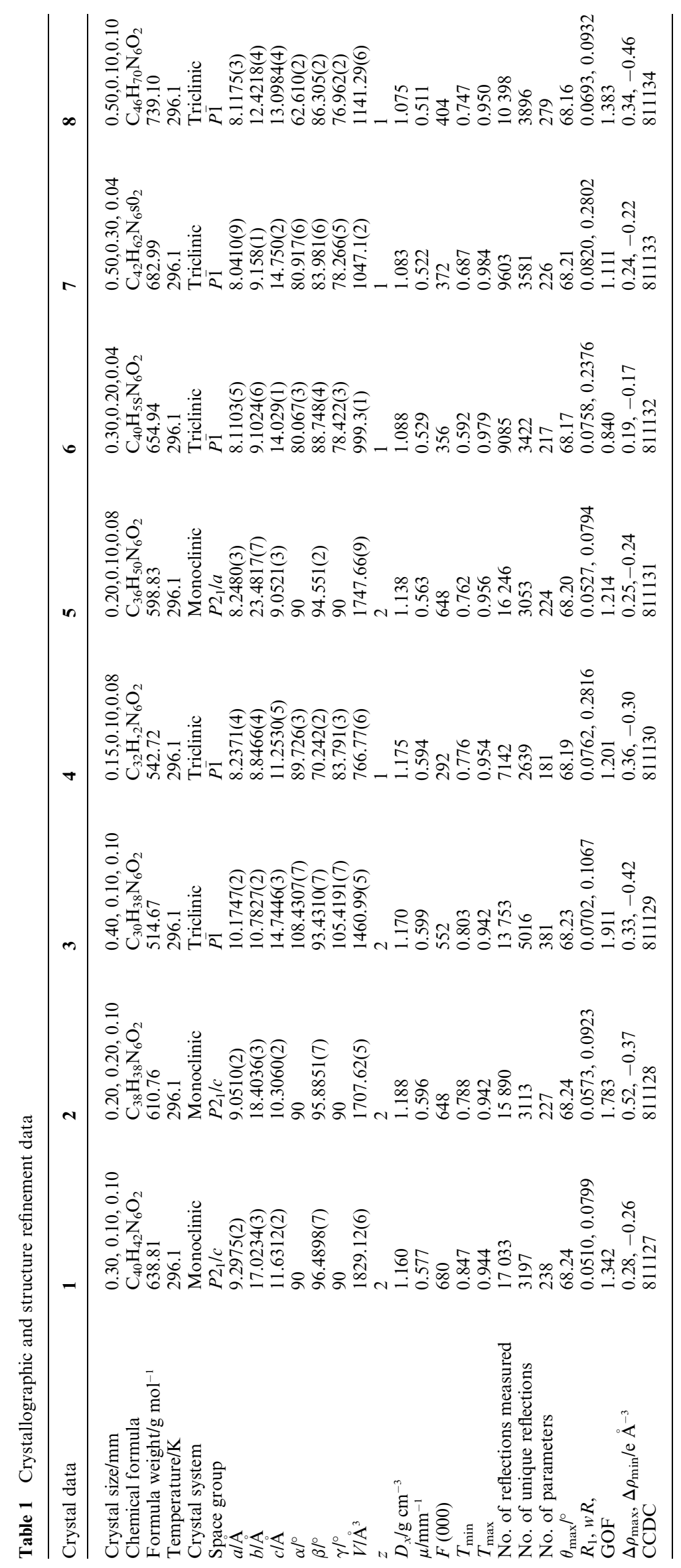




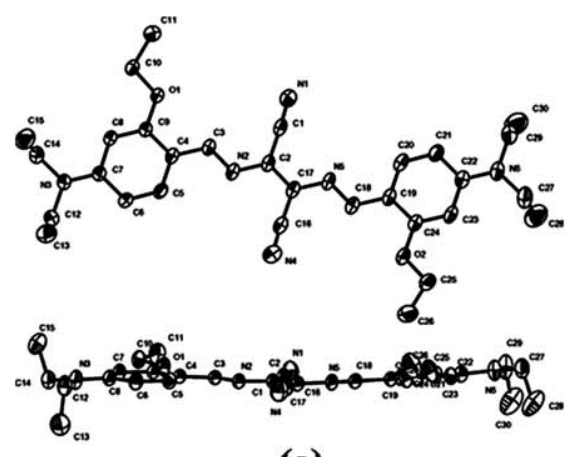

(a)

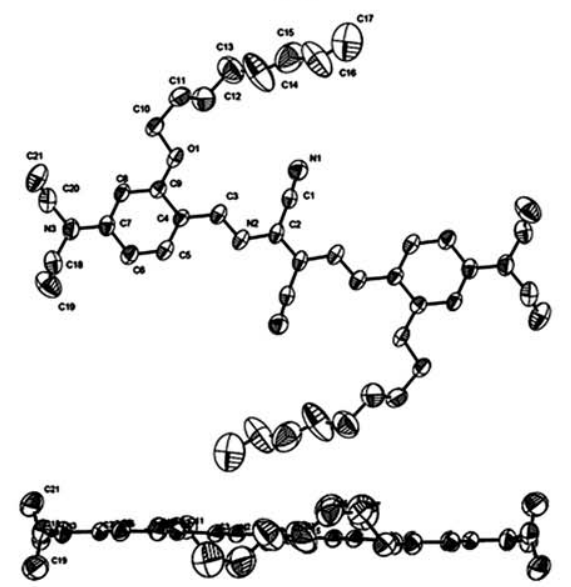

(b)

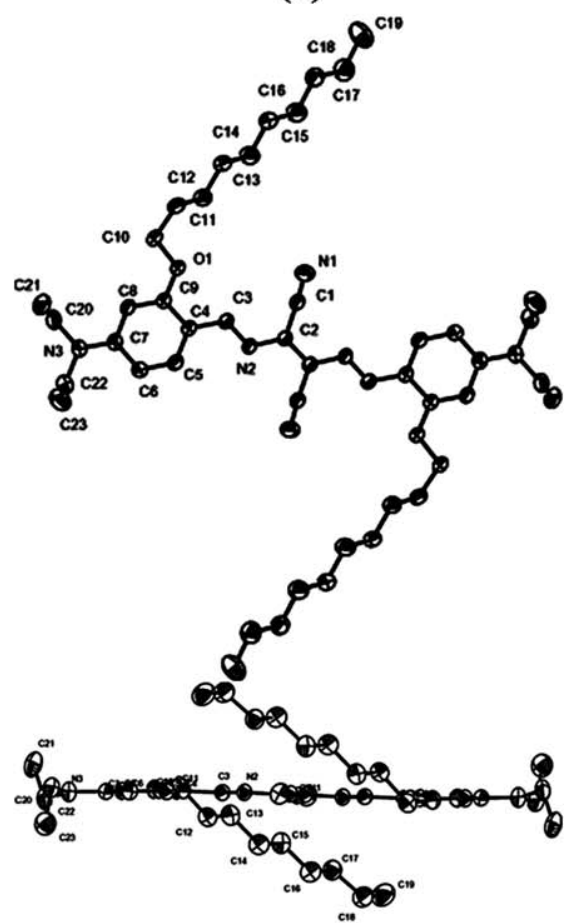

(c)

Fig. 1 Molecular structures (ORTEP) of dyes 3 (a), 7 (b) and 8 (c) drawn with $30 \%$ ellipsoidal probability; $\mathrm{H}$ atoms are omitted for clarity.

X-ray diffraction of a LB-film of a merocyanine dye. ${ }^{16}$ His group proved that the characteristic optical properties of J-aggregates are caused by the electronic states, based on the proposed
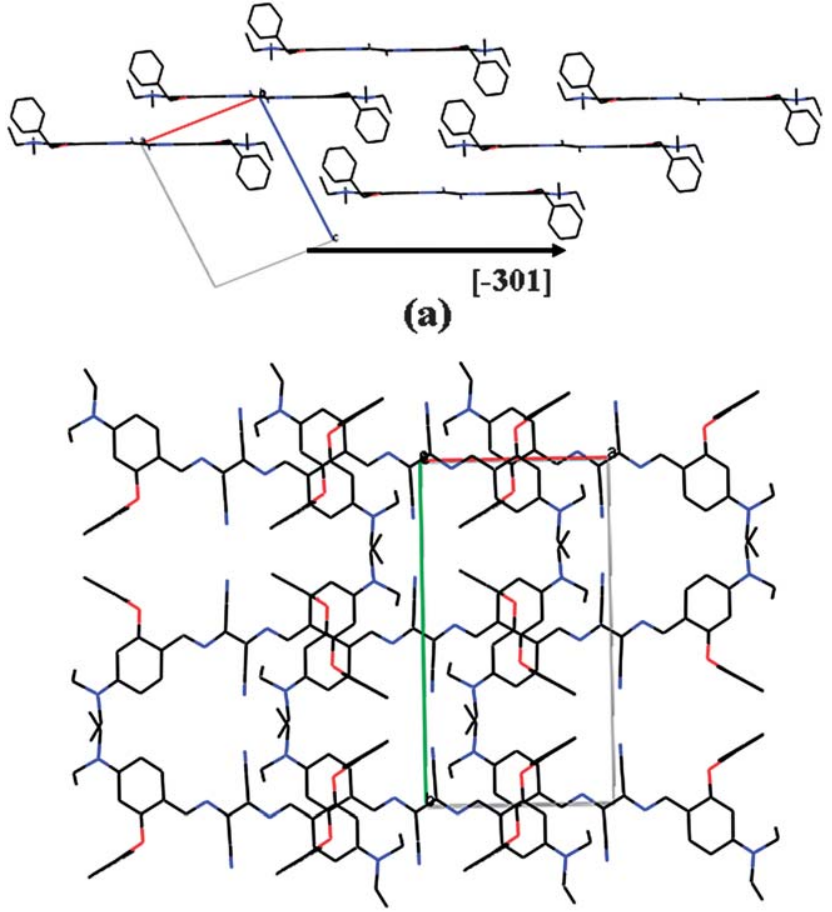

(b)

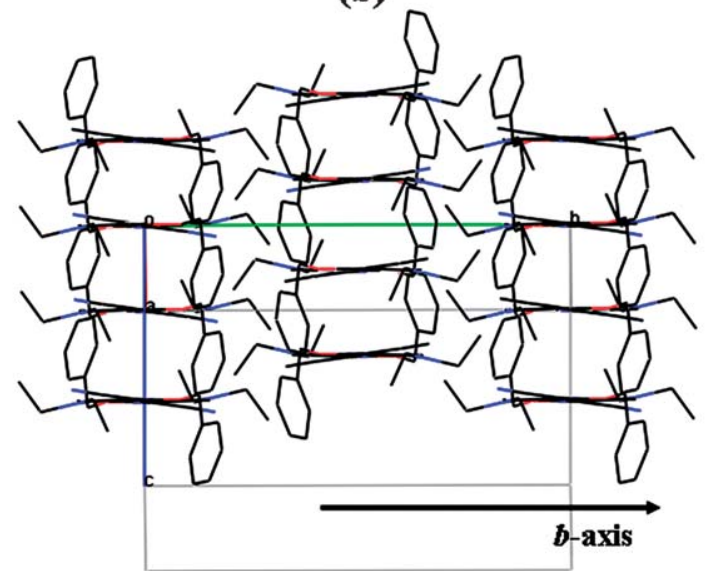

(c)

Fig. 2 Molecular arrangements of dye 1: (a) on the (103) plane, (b) looking down the normal to the (103) plane, and (c) looking down the [301] direction.

brick-wall 2-D molecular arrangement, by theoretical estimation using X-ray data. $\mathrm{Smith}^{28}$ and $\mathrm{Nakatsu}^{29}$ separately reported crystal structure studies of cyanine dyes, focusing on low-D molecular arrangements in the crystal structures. They suggested that the cyanine dyes have the potential for J-aggregate formation because of the constituent molecular arrangements in their crystal structures. However, the question of how we can design crystalline 3-D J-aggregates has not yet been resolved, although there have been many publications on the formation of J-aggregate solids on crystal surfaces, ${ }^{30,31}$ in vapour-deposited films on glass substrates, ${ }^{32-36}$ and as thin-needle solids. ${ }^{37,38}$

A bisazomethine dye, $N, N^{\prime}$-bis[4-( $N, N$-diethylamino)benzylidene]diaminomaleonitrile (DE2), derived from diaminomaleonitrile and 4-diethylaminobenzaldehyde, has been reported 
to form $\mathrm{J}$-aggregates in vapour-deposited films on glass substrates, ${ }^{32}$ and its parent derivatives are also known to exhibit J-aggregate-like absorption bands in films. ${ }^{33,34}$ These $\mathrm{J}$-aggregate films, however, also include a non-aggregated solid-phase corresponding to a single-crystal phase. The J-aggregates of DE2 were found to be meta-stable phases, ${ }^{32}$ and morphological studies revealed that the aggregates are formed on the film surface. ${ }^{33}$

In contrast, crystal structure studies on some bisazomethine dyes $^{39-41}$ showed that the crystal structure of this dye system can be fractionalized into the proposed low-D structural units of J-aggregates. Their crystal structure is characterized by a 2-D molecular stacking layer in which molecules are $\pi-\pi$ stacked in a staircase arrangement with small slip angles along the long molecular axis. ${ }^{39}$ The optical transition moment for visible absorption in this dye system is calculated to be on the molecular plane, almost in parallel with the long molecular axis. ${ }^{42}$ The observed staircase stacking arrangement is thus regarded as a potential $\mathrm{J}$-aggregate structure. This may indicate that this dye

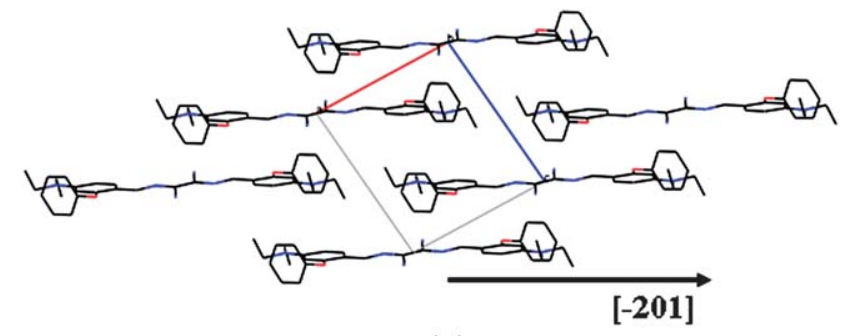

(a)

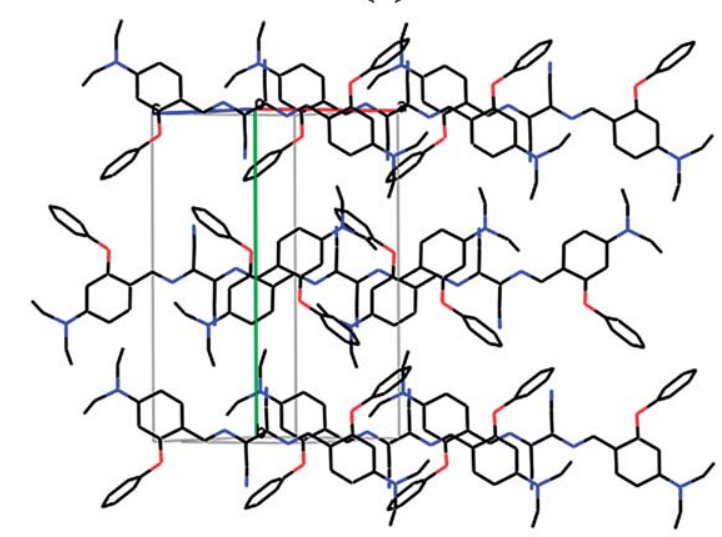

(b)

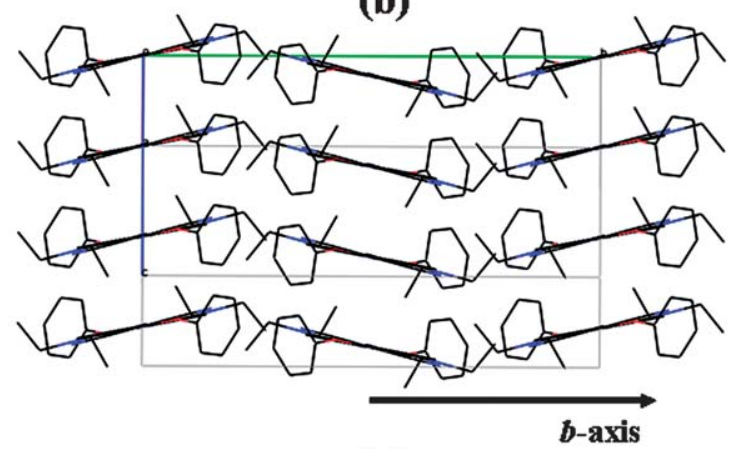

(c)

Fig. 3 Molecular arrangements of dye 2: (a) on the (102) plane, (b) looking down the normal to the (102) plane, and (c) looking down the [201] direction. system has the structure needed for potential formation of J-aggregates, as Smith and Nakatsu described in their reports. ${ }^{28,29}$ The 2-D stacking layers are further aligned along the short molecular axis to form a 3-D crystal structure. With respect to the molecular arrangement along the short molecular axis, the cyano group, the substituents on the phenyl rings $\left(\mathrm{R}_{1}\right)$, and the alkyl substituents on the amino groups $\left(R_{2}\right)$ are related to the spacing between the adjacent 2-D stacking layers.

This spacing between neighbouring layers is regarded as one of the important parameters for gaining pure J-aggregate solid films in this dye system because the physico-chemical properties of $\mathrm{J}$-aggregates are considered to be based on a low-D electronic state.

In this study, our attention was focused on substituent effects on the molecular arrangements in a series of bisazomethine dyes. Among the substituents, $R_{1}$ in particular was examined, focusing on the geometry of the 2-D stacking layers and the spacing between the stacking layers. In this study, the terminal amino group is a diethylamino moiety. The effects of $\mathrm{R}_{1}$ were investigated by analyzing the crystal structures of the dyes in detail, and were also interpreted using lattice energy calculations.

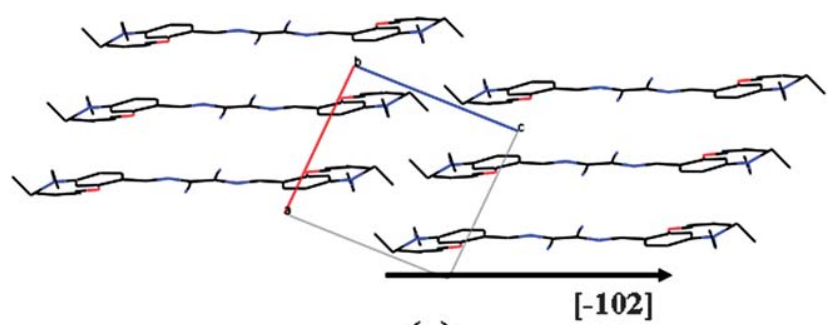

(a)

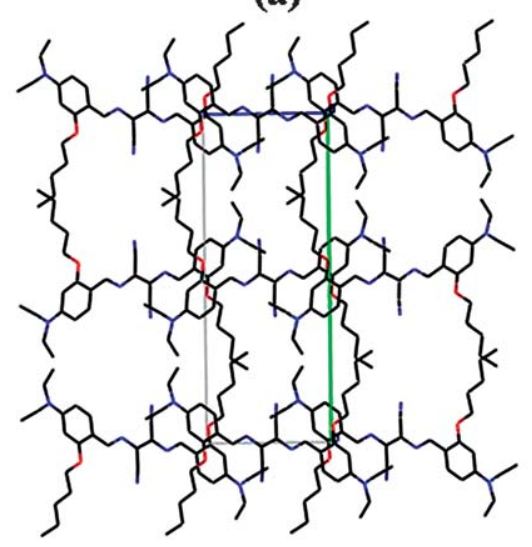

(b)

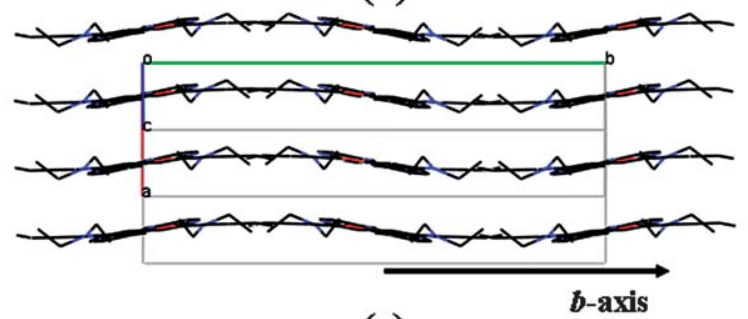

(c)

Fig. 4 Molecular arrangements of dye 5: (a) on the (201) plane, (b) looking down the normal to the (201) plane, and (c) looking down the [102] direction. 


\section{Experimental}

The bisazomethine dyes 1-8 (Scheme 1) were synthesized by the reported procedure ${ }^{43}$ and characterized by conventional analytical methods. The synthesis and optical properties in solution and in vapour-deposited films of these dyes have been published. ${ }^{42}$

\subsection{Crystal growth and data collection}

Purple crystals of $\mathbf{1 - 3 , 5}$, and $\mathbf{6}$, as well as reddish purple crystals of $\mathbf{7}$ and $\mathbf{8}$, suitable for X-ray diffraction measurements, were grown by a solvent-diffusion method. Chloroform and dichloromethane were used as good solvents for 1-3 and 5-8, respectively. $n$-Hexane and $n$-pentane were used as poor solvents for 1-6, 8, and 7, respectively. Purple crystals of $\mathbf{4}$ were obtained by slowly evaporating its chloroform solution at room temperature. X-Ray diffraction experiments were carried out with

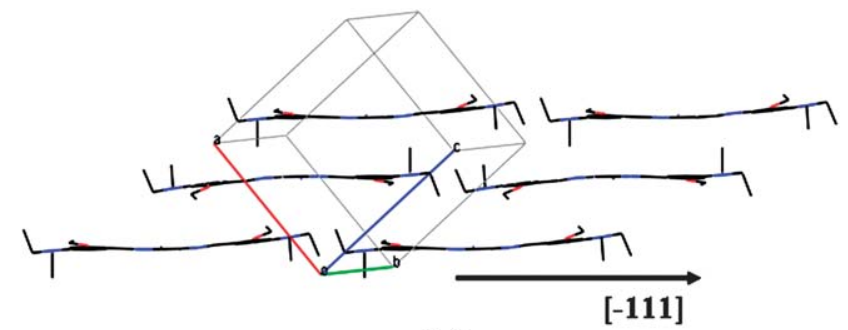

(a)

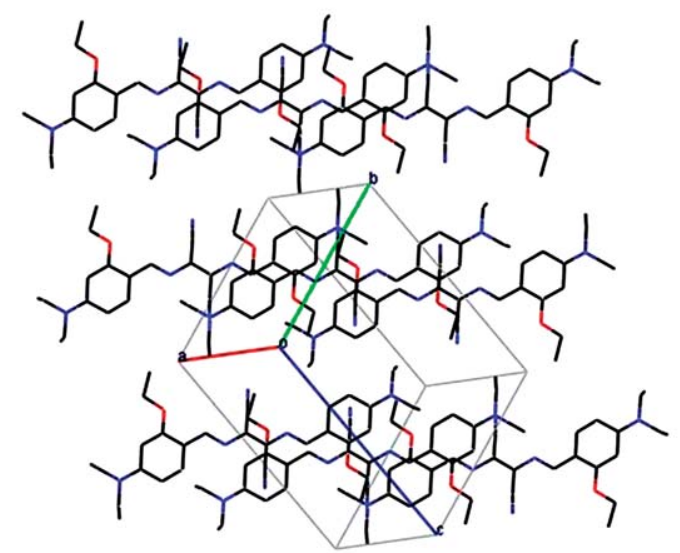

(b)

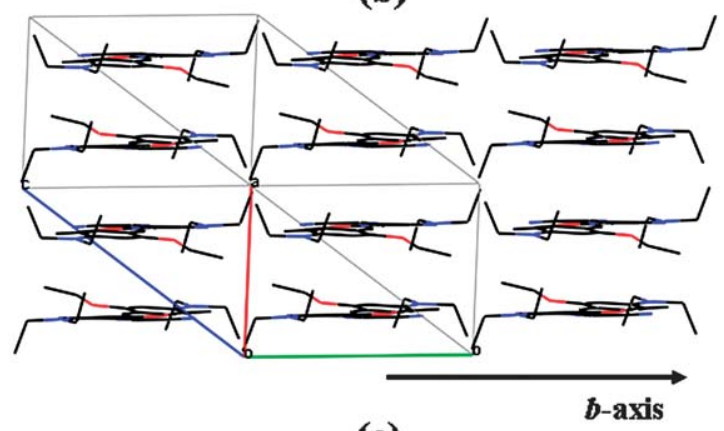

(c)

Fig. 5 Molecular arrangements of dye 3: (a) on the (202) plane, (b) looking down the normal to the (202) plane, and (c) looking down the [1111] direction. a Rigaku RAXIS-RAPID imaging plate diffractometer with graphite monochromated $\mathrm{Cu} K_{\alpha}$ radiation at $40 \mathrm{kV}$ and $30 \mathrm{~mA}$ at room temperature. The structures were solved by direct methods: SIR $92^{44}$ for 1-5, SIR $2004^{45}$ for $\mathbf{6}$ and 7, and SHELXS $97^{46}$ for $\mathbf{8}$; they were refined by full-matrix least-squares calculations. Nonhydrogen atoms were refined with anisotropic displacement. The positions of all the hydrogen atoms were geometrically calculated and refined by the riding model. All calculations were performed using the CrystalStructure $3.8^{47}$ and $4.0^{48}$ crystallographic software package. The packing diagrams were drawn using the Mercury 1.4.2. ${ }^{49}$

\subsection{Lattice energy calculations}

Lattice energy calculations were performed using the ZipOpec module, which calculates lattice energies, packing coefficients, short atom-atom distances, and significant molecule-molecule distances, using the known cell dimensions, space groups, and atomic coordinates for crystal and empirical atom-atom force

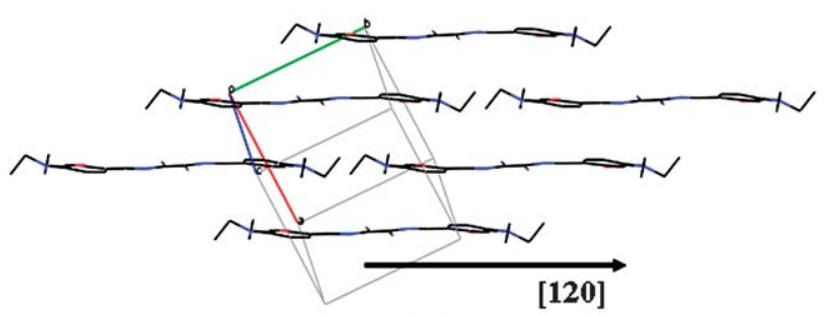

(a)

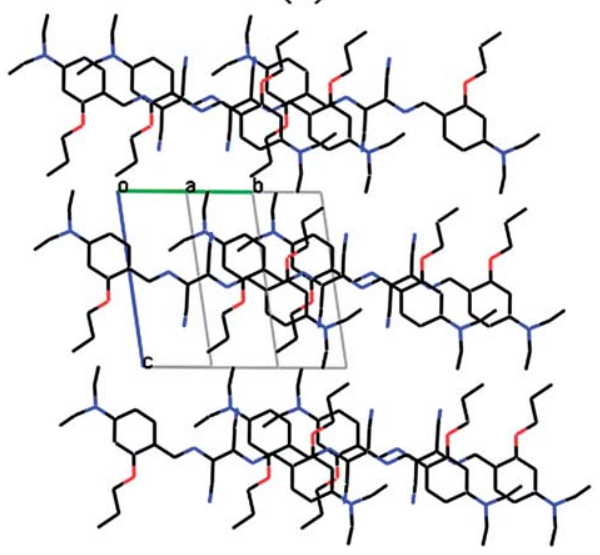

(b)

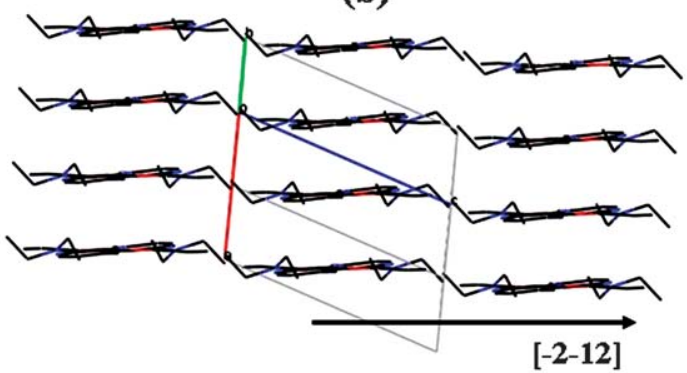

(c)

Fig. 6 Molecular arrangements of dye 4: (a) on the (21) plane, (b) looking down the normal to the (21) plane, and (c) looking down the [120] direction. 
fields (UNI potentials) ${ }^{50}$ of the OPiX program suite. ${ }^{51,52}$ The functional form of the $i-j$ atom-atom potential was calculated by the following formula: ${ }^{53}$

$$
E\left(i j, \mathrm{~kJ} \mathrm{~mol}^{-1}\right)=A \exp \left(-B R_{i j}\right)-C R_{i j}{ }^{-6}+q_{i} q_{j} / R_{i j}
$$

where $R_{i j}$ is the intermolecular distance between atomic nuclear positions, and the coefficients $A, B$, and $C$, and $q_{i}$ and $q_{j}$ denote empirical parameters and atomic charge parameters located at an atomic nuclear position, respectively. The total lattice energy is the lattice sum over $i$ and $j$. Calculations were performed using $\mathrm{X}$-ray crystal atomic coordinates. The output from these calculations yields the total energy and the contribution of molecular pairs to the lattice energy.

\section{Results and discussion}

\subsection{Molecular structure}

Details of crystal data collection and structure refinement parameters for all the dyes are listed in Table 1 and the ORTEP

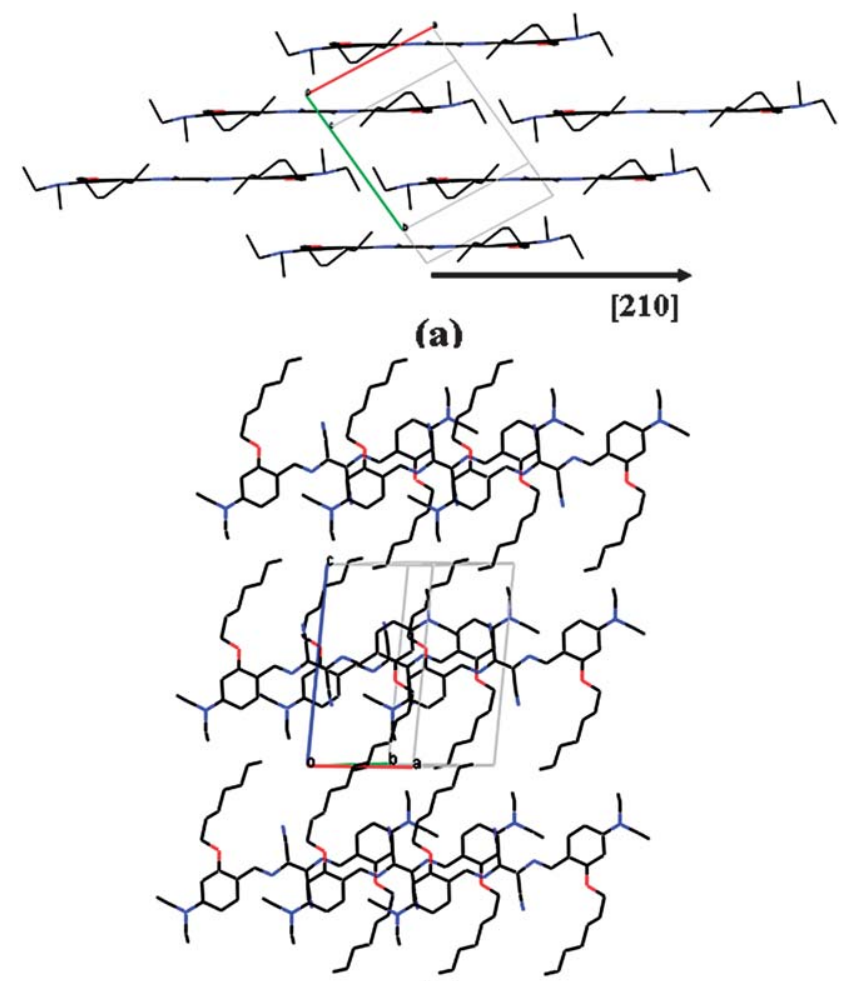

(b)

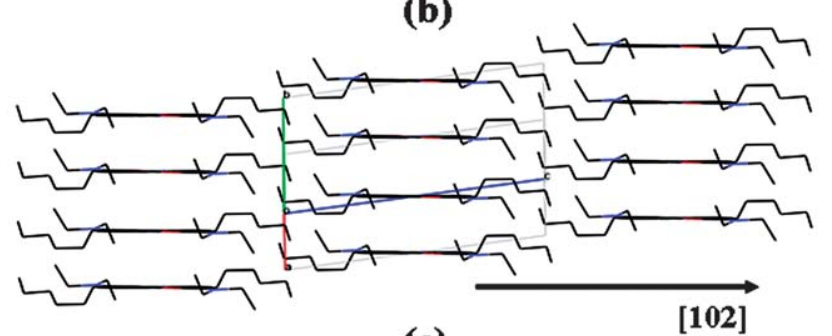

(c)

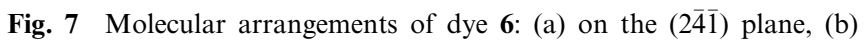
looking down the normal to the $(2 \overline{4} \overline{1})$ plane, and (c) looking down the [210] direction. drawings of molecular structures for three representative dyes are given in Fig. 1.

Seven of the eight derivatives have an inversion symmetry in their molecular structures (Fig. 1 (b)). This is consequently reflected in the conformation of the central ethylene unit, giving a trans structure. Only 3 has no inversion symmetry in the molecular structure (Fig. 1 (a)). The resulting difference in the structure from that of the other symmetric derivatives was recognized in the conformation of the terminal diethylamino groups, while the central ethylene unit has a trans conformation. In 3 , the two terminal amino groups displayed a different structural combination. One amino moiety has a staggered conformation, and the two ethyl substituents are projected in the same direction in the other amino moiety. Such a variety of structural combinations of the terminal diethyl substituents may suggest their potential for structural disorder in solids, as observed in the structural analysis of DE2. ${ }^{39}$

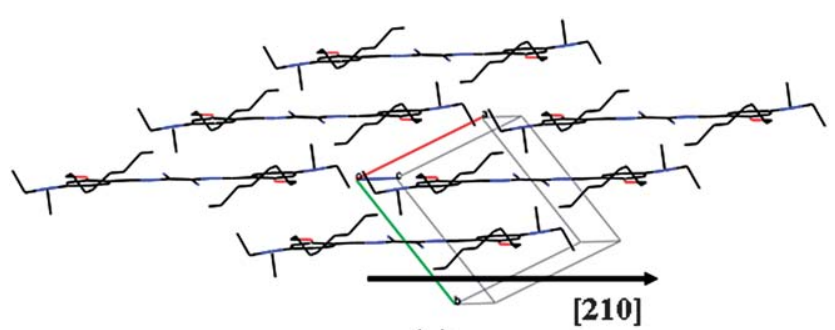

(a)

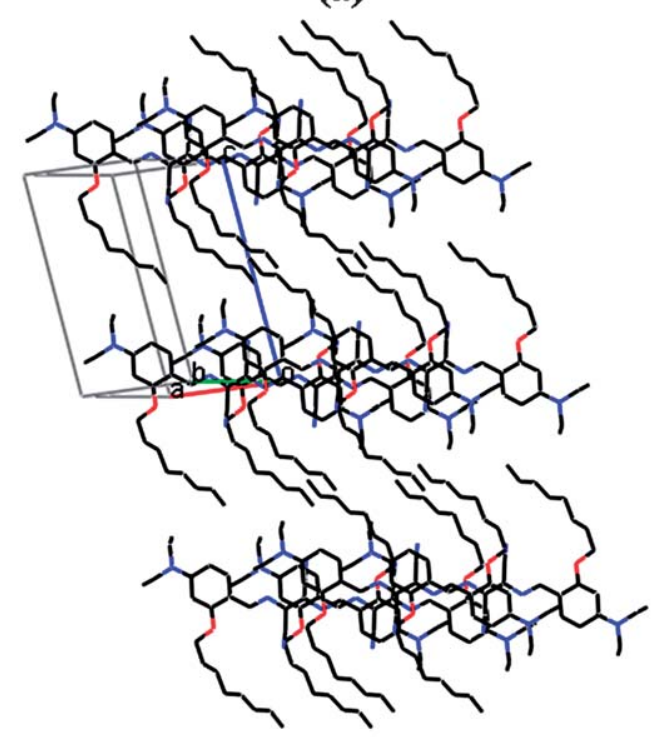

(b)

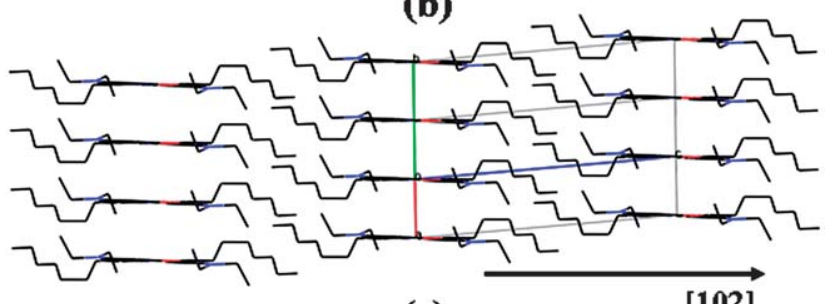

(c)

[102]

Fig. 8 Molecular arrangements of dye 7: (a) on the (120) plane, (b) looking down the normal to the (120) plane, and (c) looking down the [210] direction. 


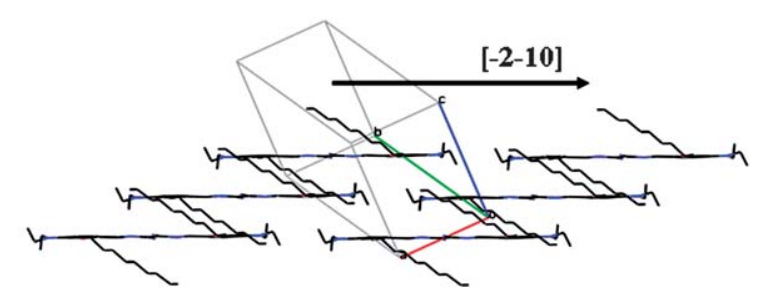

(a)

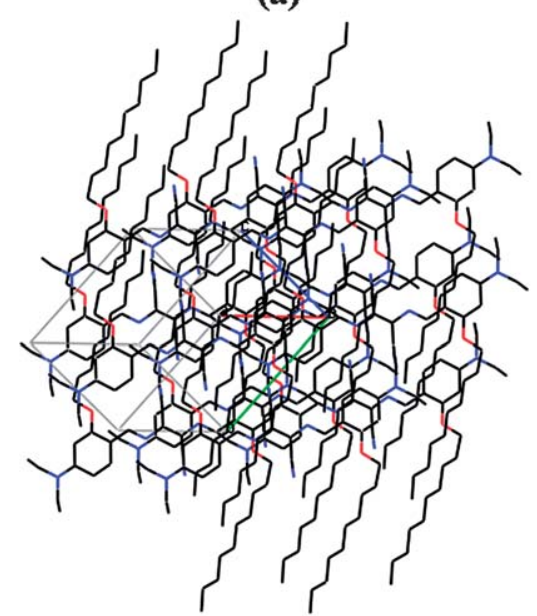

(b)

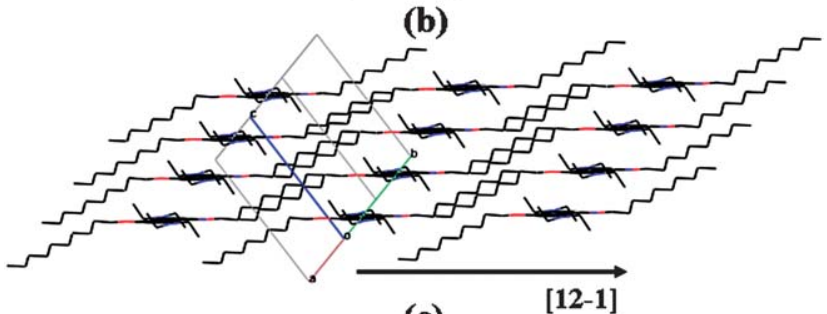

(c)

$[12-1]$

Fig. 9 Molecular arrangements of dye 8: (a) on the (1 $\overline{2} \overline{3})$ plane, (b) looking down the normal to the $(1 \overline{2} \overline{3})$ plane, and (c) looking down the $[\overline{2} \overline{1} 0]$ direction.

The geometry of $R_{1}$ was classified into two types. In $2-7$, the substituents are located on the molecular plane, whereas $\mathbf{1}$ and $\mathbf{8}$ have a conformation in which the substituents are projected out of the molecular plane (Fig. 1(c)). Little bond alternation was seen in all the dyes, and this clearly indicates that effective $\pi$ conjugation is developed between the electro-donating amino groups and the electro-accepting dicyanoethylene groups. This should reflect an almost planar molecular plane with a mean deviation of the component atoms of the chromophoric system in the range of $0.002 \AA$ from $0.234 \AA$.

Table 2 Distances between stacking molecules and the interlayer distances between adjacent 2-D layers in dyes 1-8

\begin{tabular}{|c|c|c|c|c|c|c|c|c|}
\hline & 1 & 2 & 3 & 4 & 5 & 6 & 7 & 8 \\
\hline $\begin{array}{l}\text { Stacking molecular } \\
\text { distance/Å }\end{array}$ & 3.48 & 4.38 & 3.78 & 3.52 & 3.56 & 3.63 & 3.63 & 3.60 \\
\hline $\begin{array}{l}\text { Interlayer distance } \\
\text { between adjacent 2-D } \\
\text { layers/̊̊ }\end{array}$ & 8.51 & 9.21 & 9.84 & 10.58 & 11.74 & 13.80 & 14.521 & 11.59 \\
\hline
\end{tabular}

\subsection{Crystal structures}

Single-crystals of $\mathbf{1}, \mathbf{2}$, and $\mathbf{5}$ belong to the monoclinic crystal system, with the same space group irrespective of the different substituents. Structural similarities are also seen in their molecular arrangements. It is also interesting that the geometry of their crystal lattices and the geometry of their molecular arrangements harmonized with each other. Their molecules are $\pi-\pi$ stacked in a layer parallel to the $a c$ plane, and the molecules are aligned in parallel with the long molecular axis. As illustrated in Fig. 2(a), 3(a), and 4(a), for 1, 2, and 5, respectively, the molecular arrangements in these stacking layers are identified with the staircase arrangement which is one of the proposed 2-D molecular arrangements for J-aggregates. ${ }^{28,29}$ These 2-D stacking layers are further aligned along the $b$-axis to form the crystal structure. The direction of the short molecular axis is conformable with the $b$-axis, and thus the $\mathrm{R}_{1}$ groups are considered to play a major role in the separation of the 2-D stacking layers. The two slip angles between the stacking molecules are estimated to be $9^{\circ}$ and $21^{\circ}$ for $1,21^{\circ}$ and $23^{\circ}$ for 2 , and $16^{\circ}$ and $24^{\circ}$ for $\mathbf{5}$. A large transition moment corresponding to intense visible absorption is known to lie on the $\pi$-plane along the long molecular axis in this dye system. ${ }^{42}$ Their estimated small slip angles therefore imply that their 2-D stacking layers can be regarded as potential structural components for J-aggregates. ${ }^{25}$

Dyes 3, 4, 6, 7, and 8 were found to crystallize in a different crystal system from that of $\mathbf{1}, \mathbf{2}$, and $\mathbf{5}$. As illustrated in Fig. 5, 6, 7 , and 8 for $\mathbf{3}, \mathbf{4}, \mathbf{6}$, and 7 , respectively, the geometries of their molecular arrangements were identified as being similar to those of $\mathbf{1}, \mathbf{2}$, and $\mathbf{5}$. In 3, 4, 6, and 7 similar 2-D staircase stacking layers were observed and the 3-D crystal structure is formed by aligning these 2-D layers along the short molecular axis. Within the staircase stacking layer, molecules are stacked along the long molecular axis with small slip angles of $18^{\circ}$ and $23^{\circ}$ in $3,16^{\circ}$ and $24^{\circ}$ in $4,16^{\circ}$ and $25^{\circ}$ in 6 , and $16^{\circ}$ and $26^{\circ}$ in 7 . The spacing between the 2-D layers is occupied by $R_{1}$. This spatial filling feature is basically equivalent to that for $\mathbf{1}, \mathbf{2}$, and $\mathbf{5}$. Although the symmetry of the molecular conformation of $\mathbf{3}$ is different, it was found to crystallize in the same manner. This might indicate that the difference in conformation at the terminal group has no significant impact on the formation of the crystal structure in the diethylamino series of this dye system. These seven dyes were classified into two different crystal systems. However, their space filling feature in the crystal structure was identified as being the same.

However, in $\mathbf{8}$, which has a long $\mathrm{C} 10$ alkyl chain on the phenyl rings, the above structural similarity was not clearly seen. As shown in Fig. 9, the molecules were arranged in the staircase fashion with a slight dislocation along the short molecular axis. The decyl chains have a non-planar conformation in which the eight carbon atoms of the chain have a typical zigzag conformation projected out of the molecular $\pi$-plane. These projected segments of the alkyl chains are packed with a gradient angle of about $59^{\circ}$ between the molecular $\pi$-plane and the alkyl chains, and are directed in the $[1 \overline{1} 0]$ direction. As a result of the close packing nature adopted by crystals to minimize their lattice energy, the long alkyl chains formed these spatial conformations and, consequently, the molecular stacking arrangement underwent a significant change. The structural difference between $\mathbf{8}$ 
Table 3 Results of lattice energy calculations for dyes $\mathbf{1}-\mathbf{8}^{a}$

\begin{tabular}{|c|c|c|c|}
\hline & $\begin{array}{l}\text { Lattice } \\
\text { energy } / \mathrm{kJ} \mathrm{mol}^{-1}\end{array}$ & \multicolumn{2}{|l|}{ Contribution of molecular pairs to lattice energies } \\
\hline 2 & -569.4 & $-106.1(18.6 \%, 45501$ and 65501$)$ & $-56.7(9.9 \%, 45601$ and 65401$)$ \\
\hline 3 & -525.9 & $-120.9(22.9 \%, 66502)$ & $-71.4(13.5 \%, 57602)$ \\
\hline 4 & -574.7 & $-119.8(20.8 \%, 54501$ and 56501$)$ & $-86.4(15.0 \%, 44501$ and 66501$)$ \\
\hline 5 & -596.4 & $-116.9(19.6 \%, 55401$ and 55601$)$ & $-88.6(14.8 \%, 45601$ and 65401$)$ \\
\hline 8 & -724.1 & $-140.4(19.3 \%, 45501$ and 65501$)$ & $-62.6(8.6 \%, 46401$ and 64601$)$ \\
\hline
\end{tabular}

and the other derivatives is therefore considered to reflect the difference in the energetic contribution of $\mathrm{R}_{1}$ to the whole lattice energy. This result suggested the limit of space adjustment between the 2-D stacking layers by changing the length of $R_{1}$.

Table 2 summarizes the distances between the stacking molecules and the interlayer distances between the adjacent stacking 2-D layers. Scheme 2 indicates the crystal structure of dye 7 with these two geometrical parameters.

The stacking molecular distances were observed to be around $3.5 \AA$, except for 2 . The stacking molecular distance in $\mathbf{2}$, about $4.3 \AA$, was larger than that in the other derivatives. This may be attributed to the inflexible characteristics of the phenyl group of the phenoxy substituents because, in benzyl-substituted derivative 1, the phenyl rings of the benzyloxy substituents occupied the space in such a way that they are placed away from the stacking between the planer molecular planes, as clearly indicated in Fig. 2. The interlayer distances between the adjacent 2-D stacking layers for all the dyes were estimated using the Mercury software 1.4.2. Fifty-four component atoms related to the chromophoric system of nine stacking molecules in one 2-D stacking layer were considered as a representative stacking layer in calculation of the plane. The estimated results showed that the interlayer distance between adjacent 2-D stacking layers changed from $8.51 \AA$ to $14.52 \AA$, depending on the size of $\mathrm{R}_{1}$, except for $\mathbf{8}$. Dye 8 exhibited an interlayer distance of about $11.59 \AA$ because the long alkyl substituents deviated from the molecular $\pi$-plane.
Dye 8 demonstrated the limit of the length of $R_{1}$ for spatial control between the 2-D stacking layers to retain the 2-D stacking structure with no slippage along the short molecular axis.

\subsection{Lattice energy}

In order to interpret the substituent effects from an energetic perspective, lattice energy calculations were carried out on the basis of crystal structures. Table 3 indicates the important molecular pairs in lattice formation. The first and second energetic contributions to the lattice energy were from a stacking molecular pair characterized by a large slip angle, except in the case of $\mathbf{8}$. For 1-7, stacking pairs having small slip angles were the third and fourth contributors. These results clearly indicate that the 2-D stacking arrangement has a significant impact on crystallization, and the present strategy of controlling the spacing between adjacent 2-D stacking layers by changing the substituents on the phenyl rings operates efficiently in this dye system. In the case of $\mathbf{3}$, there is no inversion symmetry in the molecular structure and this is reflected by a small difference in the third and fourth energy values. The calculated results also revealed that the symmetry difference in the molecular conformation in $\mathbf{3}$ has no significant influence on the basic skeleton of the crystal structure of this dye system. However, as the structural data and the calculation result for $\mathbf{8}$

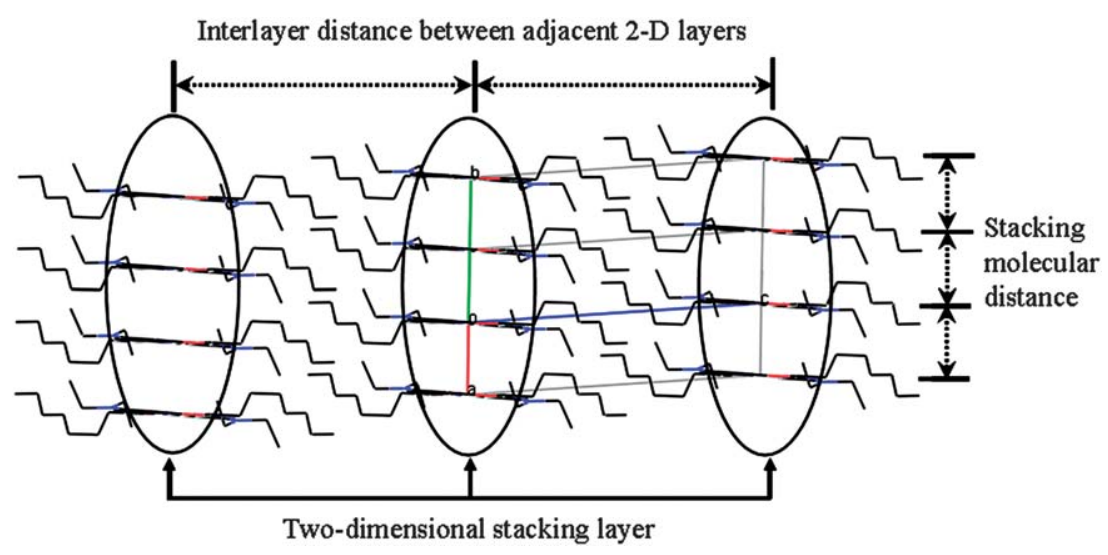

Scheme 2 Intermolecular distances between stacking molecules and the interlayer distances between adjacent 2-D layers. 
showed, the chain length of the alkoxy substituent affects the construction of a crystal lattice. In $\mathbf{8}$, the molecules are no longer stacked in the same manner as in the other dyes. The molecules are stacked with small slippages along both the long and the short molecular axes to give a skewed stacking motif in the 2-D stacking layer. The most-overlapped stacking molecular pair gave the first and second energetic contributions. The long alkyl chains projected from the planar conjugated system are stacked in parallel to the skew direction of the $\pi$-stacking molecules. This structural feature was successfully explained by the calculation results. The third and fourth energetic contributions were given by the molecular pair with ADC 46401 and 64601, in which the long alkyl chains of the alkoxy substituent are stacked with each other. This is the specific molecular pair that characterizes the crystal structure of $\mathbf{8}$. The present design strategy in this dye system, that is controlling the spacing between 2-D molecular stacking layers by the size of $R_{1}$, was found to be effective when the length of the alkyl chain was less than $\mathrm{C} 10$. This is a fundamental result for producing solid-state $\mathrm{J}$-aggregates and further consideration should be given to the effects of other structural parameters, such as the alkyl chain length of the amino group, on the crystal structure. Lattice energy calculation was conducted for the interpretation of effects of a change in molecular structure on the crystal structure in this study and the calculation result was in good agreement with their structural features for all dyes.

\section{Conclusions}

To achieve our final goal of obtaining pure J-aggregate solid films, we investigated the effects of phenyl-ring substitution on the crystal structures of a bisazomethine dye system, focusing on their 2-D molecular stacking structures and arrangements. All the dyes were found to have their molecules $\pi-\pi$ stacked in the 2-D molecular layers; these layers were characterized by a staircase arrangement. We also found that the interlayer distance between adjacent 2-D layers can be controlled by the substituents on the phenyl rings when the chain length of the substituent is less than $\mathrm{C} 10$. When the chain length is $\mathrm{C} 10$, the stacking structure in the 2-D layer changed to a staircase arrangement with a slight dislocation along the short molecular axis, and additional molecular stacking was found to form between the long alkyl chains. These structural characteristics were interpreted by considering the energetic relationship between the molecular and crystal structures on the basis of lattice energy calculations. These observations led us to conclude that, in this dye system, the substituents on the phenyl rings can be used as practical parameters in crystal engineering to obtain a quasi-lowD electronic state. Further investigations, which involve crystal structure studies on the effects of the alkyl substituents in the terminal amino groups, will be reported elsewhere.

\section{Acknowledgements}

This research was financially supported by the Japan Society for the Promotion of Science (JSPS) and the Korea Science and Engineering Foundation (KOSEF) under the Japan-Korea Basic Scientific Cooperation Program, as well as a Sasagawa Scientific Research Grant from the Japan Science Society.

\section{References}

1 K. Walzer, B. Maenning, M. Preiffer and K. Leo, Chem. Rev. (Washington, DC, U. S. ), 2007, 107, 1233-1271.

2 H. H. Sung, I. H. Kim, K. N. Byun and H. S. Yoo, Curr. Appl. Phys., 2005, 5, 345-347.

3 W. Y. Wong and C. L. Ho, J. Mater. Chem., 2009, 19, 4457-4482.

4 N. R. Armstrong, W. Wang, D. M. Alloway, D. Placencia, E. Ratcliff and M. Brumbach, Macromol. Rapid Commun., 2009, 30, 717731.

5 C. D. Dimitrakopoulos and P. R. L. Malenfant, Adv. Mater., 2002, 14, 99-117.

6 J. Zaumseil and H. Sirringhaus, Chem. Rev. (Washington, DC, U. S.), 2007, 107, 1296-1323.

7 H. Shang, H. Fan, Q. Shi, S. Li, Y. Li and X. Zhan, Sol. Energy Mater. Sol. Cells, 2010, 94, 457-464.

8 A. Hadipour, B. D. Boer and P. W. M. Blom, Adv. Funct. Mater., 2008, 18, 169-181.

9 Q. Bricaud, A. Cravino, P. Leriche and J. Roncali, Synth. Met., 2009, 159, 2534-2538.

10 H. N. Lee, Y. G. Lee, I. H. Ko, E. C. Hwang and S. K. Kang, Curr. Appl. Phys., 2008, 8, 626-630.

11 J. E. Anthony, Chem. Rev. (Washington, DC, U. S. ), 2006, 106, 50285048.

12 Y. Kumashiro, H. Nakako, M. Inada, K. Yamamoto, A. Izumi and M. Ishihara, Appl. Surf. Sci., 2009, 256, 1019-1022.

13 Y. Sun, Y. Liu and D. Zhu, J. Mater. Chem., 2005, 15, 53-65.

14 M. Matuumoto, J. Photochem. Photobiol., A, 2003, 158, 199-203.

15 K. Ikegami and M. Lan, Colloids Surf., A, 2005, 257-258, 143148.

16 N. Kato, K. Yuasa, T. Araki, I. Hirosawa, M. Sato, N. Ikeda, K. I. Iimura and U. Uesu, Phys. Rev. Lett., 2005, 94, 136404.1136404.4.

17 M. Wang, G. L. Silva and B. A. Armitage, J. Am. Chem. Soc., 2000, 122, 9977-9986.

18 S. I. Kuroda, Adv. Colloid Interface Sci., 2004, 111, 181-209.

19 T. Kobayashi, J-aggregates, World Scientific Publishing, Singapore, 1996.

20 D. Möbius, Adv. Mater., 1995, 7, 437-444.

21 K. Ikegami, J. Chem. Phys., 2004, 121, 2337-2347.

22 J. Moll, S. Daehne, J. R. Durrant and D. A. Wiersma, J. Chem. Phys., 1995, 102, 6362-6370.

23 L. S. Pu, Curr. Appl. Phys., 2001, 1, 367-370.

24 A. S. Davydov, Theory of Molecular Excitons, McGraw-Hill, New York, 1962.

25 V. Czikkely, H. D. Forsterling and H. Kuhn, Chem. Phys. Lett., 1970, 6, 11-14.

26 G. Scheibe, Angew. Chem., 1939, 52, 631-637.

27 T. Kato, F. Sasaki, S. Abe and S. Kobayashi, Chem. Phys., 1998, 230, 209-221.

28 D. L. Smith, Photogr. Sci. Eng., 1974, 18, 309-322.

29 K. Nakatsu, H. Yoshioka and S. Nishigaki, Nihon Shashin Gkkai-shi (in Japanese), 1983, 46, 89-98.

30 D. L. Smith, Photogr. Sci. Eng., 1972, 16, 329-333.

31 C. Reich, W. D. Pandolfe and G. R. Bird, Photogr. Sci. Eng., 1973, 17, 34-342.

32 S. Matsumoto, T. Kobayashi, T. Aoyama and T. Wada, Chem. Commun., 2003, 1910-1911.

33 S. Matsumoto, M. Satoh, T. Kobayashi, T. Aoyama and T. Wada, Trans. Mater. Res. Soc. Jpn., 2005, 30, 345-348.

34 T. Kobayashi, S. Matsumoto, T. Aoyama and T. Wada, Thin Solid Films, 2006, 509, 145-148.

35 F. Mizutani, S. Iijima and K. Tsuda, Bull. Chem. Soc. Jpn., 1982, 55, $1295-1299$.

36 Y. Ueda and K. Nitta, Jpn. J. Appl. Phys., 2001, 40, 6951-6955.

37 H. Yao, M. Omizo and N. Kitamura, Chem. Commun., 2000, 739740.

38 E. S. Emerson, M. A. Conlin, A. E. Rosenoff, K. S. Norlamd, H. Rodriguez, D. Chin and G. R. Bird, J. Phys. Chem., 1967, 71, 2396-2403.

39 S. Matsumoto, K. Shirai, K. Kobayashi, T. Wada and M. Shiro, Z. Kristallogr., 2004, 219, 239-243.

40 S. H. Kim and S. Matsumoto, Dyes Pigm., 2007, 72, 406-408.

41 Y. A. Son, S. Matsumoto, E. M. Han, S. Wang and S. H. Kim, Mol. Cryst. Liq. Cryst., 2008, 492, 46/[410]-55/[419]. 
42 B. S. Kim, D. Kashibuchi, Y. A. Son, S. H. Kim and S. Matsumoto, Dyes Pigm., 2011, 90, 56-64.

43 K. Shirai, M. Matsuoka and K. Fukunishi, Dyes Pigm., 2000, 47, $107-115$.

44 A. Altomare, G. Cascarano, C. Giacovazzo, A. Guagliardi, M. Burla, G. Polidori and M. Camalli, J. Appl. Crystallogr., 1994, 27, 435-436.

45 M. C. Burla, R. Caliandro, M. Camalli, B. Carrozzini, G. L. Cascarano, L. D. Caro, C. Giacovazzo, G. Polidori and R. Spagna, J. Appl. Crystallogr., 2005, 38, 381-388.

46 G. M. Sheldrick, Program for the Solution of Crystal Structures, University Göttingen, Germany, 1997.

47 CrystalStructure 3.8: Crystal Structure Analysis Package, Rigaku and Rigaku/MSC, The Woodlands, TX, USA, 1998-2007.
48 CrystalStructure 4.0: Crystal Structure Analysis Package, Rigaku Corporation, Tokyo 196-8666, Japan, 2000-2010.

49 C. F. Macrae, P. R. Edgington, P. McCabe, E. Pidcock, G. P. Shields, R. Taylor, M. Towler and J. van de Streek, J. Appl. Crystallogr., 2006, 39, 453-457.

50 G. Filippini and A. Gavezzotti, Acta Crystallogr., Sect. B: Struct. Sci., 1993, 49, 868-880.

51 A. Gavezzotti, OPiX: a Computer Program Package for the Calculation of Intermolecular Interactions and Crystal Energies, University of Milan, Milan, Italy, 2003.

52 A. Gavezzotti, Z. Kristallogr., 2005, 220, 499-510.

53 A. I. Kitaigorodsky, Molecular Crystals and Molecules, Academic Press, New York, 1973. 